\title{
OPTICAL WHITENING AND BRIGHTENING Of Pearls: A Fluorescence SPECTROSCOPY STUDY
}

Chunhui Zhou, Tsung-Han Tsai, Nicholas Sturman, Nanthaporn Nilpetploy, Areeya Manustrong, and Kwanreun Lawanwong

White pearls and those with very light hues are routinely processed or treated by various methods after harvest, especially akoya and freshwater cultured pearls. Bleaching is the most prevalent and is often used in addition to maeshori treatment, an umbrella term for various types of luster enhancements. However, little reference has been made in the literature to the use of optical brighteners, the focus of this study, within the pearl trade. In this project, known samples of optically brightened akoya cultured pearls were studied and compared with their non-brightened counterparts and cultured pearls of various types. In addition, a South Sea cultured pearl was treated in-house with a commercial whitening product. Our preliminary results indicated that pearls treated by this method showed strong bluish fluorescence under long-wave ultraviolet (UV) excitation, and can be confidently separated from non-brightened pearls using fluorescence spectroscopy when a distinct fluorescence maximum at around 430 to $440 \mathrm{~nm}$ is observed.

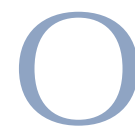
ptical brightening agents (OBAs) are chemical compounds that can absorb light in the ultraviolet and violet region of the electromagnetic spectrum and emit light in the blue region as fluorescence, due to their extended conjugation and/or aromaticity. They are sometimes called fluorescent brightening agents or fluorescent whitening agents, and have been frequently used to enhance the appearance of fabric and paper (Lanter, 1966; Leaver and Milligan, 1984; Esteves et al., 2004; Bajpai, 2018). While many types of brighteners are listed in the Colour Index (https://colour-index.com), only a handful are commercially important. Some examples are shown in figure 1.

Photoluminescence is light emission from any form of matter after the absorption of photons. Fluorescence is a type of photoluminescence in which a molecule dissipates its absorbed energy through the rapid emission of a photon, while phosphorescence is the emission of radiation in a similar manner to fluorescence but on a longer timescale, so that emission continues after excitation ceases. Fluorescence

See end of article for About the Authors and Acknowledgments.

Gems \& Gemology, Vol. 56, No. 2, pp. 258-265,

http://dx.doi.org/10.5741/GEMS.56.2.258

(C) 2020 Gemological Institute of America can be generated by exciting the substance via a range of energy sources. The molecule in the substance absorbs the source energy and once excited moves from a lower electronic state to a higher one. Immediately after absorbing energy, it loses the energy by emitting a photon; this process of photon emission is called luminescence. Typically, fluores-

\section{In Brief}

- Akoya and freshwater cultured pearls are routinely processed to improve their appearance.

- Long-wave UV radiation is a quick and useful tool to aid in the identification of some treatments.

- Optically brightened pearls appear white with the addition of agents, while bleaching makes pearls whiter by removing discoloration or pigmentation.

- Fluorescence spectroscopy is a quick and effective way of separating optically brightened pearls from bleached and untreated ones.

cence occurs at a lower energy than the absorbed radiation; this phenomenon is known as the Stokes shift (Lakowicz, 2006).

Fluorescence occurs widely in nature. For example, many kinds of gemstones such as rubies, emer- 


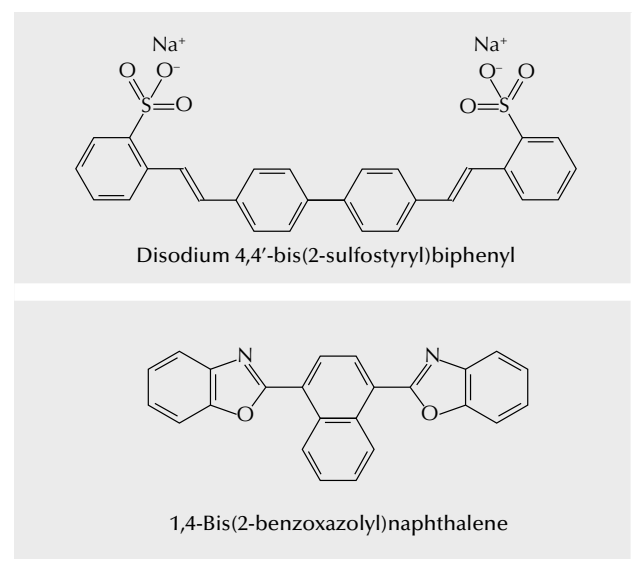

alds, and diamonds can emit visible light when exposed to ultraviolet light. For research purposes, fluorescence can be measured by fluorescence spectroscopy. The emission signal from the sample is collected by a spectrometer to monitor the distribution in fluorescence intensity as a function of wavelength. Since fluorescence has a short lifetime, typically decaying within nanoseconds after the excitation, the sample must be constantly illuminated to maintain its fluorescence signal.

Fluorescence spectroscopy has been used extensively as a basic tool in a wide range of research fields, including chemistry, biology, mineralogy, and gemology (Lakowicz, 2006). For example, structural defects in minerals usually result in characteristic fluorescence. A typical application is to use fluorescence spectroscopy to distinguish natural diamond from its synthetic counterparts (Tsai and D'Haenens-Johansson, 2019). One important feature of fluorescence spectroscopy is its high sensitivity, which enables it to detect the luminescence signal from a near-zero background. Modern sensors make single-photon sensitivity possible. As a result, fluorescence spectroscopy is extremely sensitive, allowing for the detection of weak signals. Pearl, a biogenically originated material, inherits fluorescence characteristics typical of many organic materials, and the biogenic aragonite that constitutes the bulk of a pearl is well known for its fluorescence properties. These facts make fluorescence spectroscopy a potentially very useful tool for the identification of pearls from different species located in various areas.

Since both pearls and OBAs possess characteristic fluorescence features, the use of fluorescence spectroscopy makes it possible to easily detect pearls treated by such agents. The traditional way to observe pearl fluorescence is to use long-wave UV (LWUV) and short-wave UV (SWUV) lamps to excite the sample and visually observe the fluorescence effect (color, intensity, distribution, etc.). Untreated white to cream-colored pearls generally show blue to greenish blue fluorescence under LWUV excitation. Akoya and freshwater cultured pearls, which are routinely processed, typically show strong bluish white fluorescence. Similar to these pearls, OBAs are also highly fluorescent. With exposure to UV radiation, a bright blue glow is visible from the OBA solution (an example is shown in figure 2). The use of fluorescence spectroscopy can easily separate the minor differences in fluorescence color exhibited by some pearls and the OBAs.

Figure 2. An example of blue fluorescence emitted by an OBA solution used in our in-house treatment experiment under LWUV radiation. Photo by Chunhui Zhou.

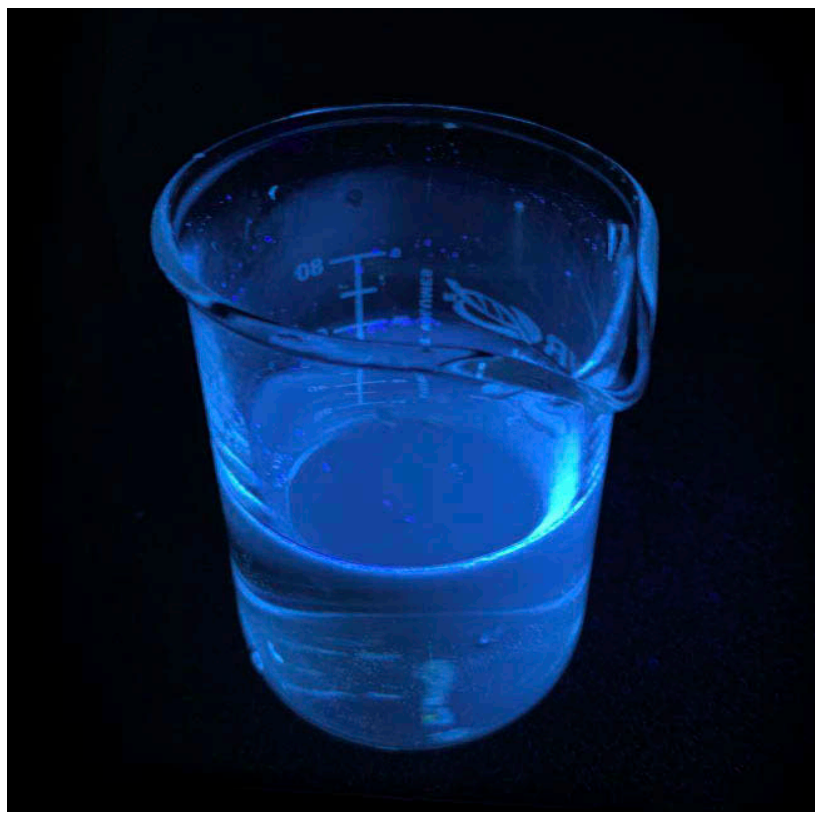


The use of OBAs in the cultured pearl industry is not unusual (Song and Fan, 1999; Japan Pearl Promotion Society, 2014), as they are sometimes routinely applied to cultured pearls post-harvest along with other types of processes such as bleaching or maeshori (which refers to various types of luster enhancement), but very little has been written in the gemological literature about the identification of this process (Shor, 2007). It is important to differentiate between the whitening effects due to bleaching and those produced by optical brightening, as they depend on two very different mechanisms: bleaching removes, or reduces the concentrations of, the color-causing pigmentations within pearls, while optical brighteners add an artificial optical effect to pearls, making them look less yellow by increasing the overall amount of blue light emitted due to these chemicals' blue fluorescence properties. The focus of this study is to use fluorescence spectroscopy as an easy method to detect the presence of OBAs. Such a technique would be of great practical interest to the trade and public in order to faithfully differentiate treated from untreated pearls.

\section{MATERIALS AND METHODS}

Two groups of mostly white-colored akoya bead cultured pearls ranging from $6.97 \times 6.54 \mathrm{~mm}$ to $8.14 \times$ $7.48 \mathrm{~mm}$ were supplied by Orient Pearl (Bangkok) Ltd. One group (139 pearls) was reportedly treated with unidentified OBAs in addition to the routine bleaching process, while the other group (150 pearls) was only bleached and not treated with OBAs (figure 3). In addition, three different types of white cultured pearls (akoya, freshwater, and South Sea) from GIA's research collection (figure 4), as well as samples from an untreated akoya strand reportedly cultured in Vietnam, also from GIA's research collection (figure 5), were included in the study.

One white South Sea cultured pearl was also treated in-house using a commercial laundry product (Rit Whitener \& Brightener). According to the label, this product contained optical brighteners and sodium chloride. The pearl was immersed in the saturated brightener solution (approximately $1 \mathrm{~g}$ of brightener powder per $100 \mathrm{~mL}$ of water) for 3 hours at a slightly elevated temperature (about $40^{\circ} \mathrm{C}$ ), with
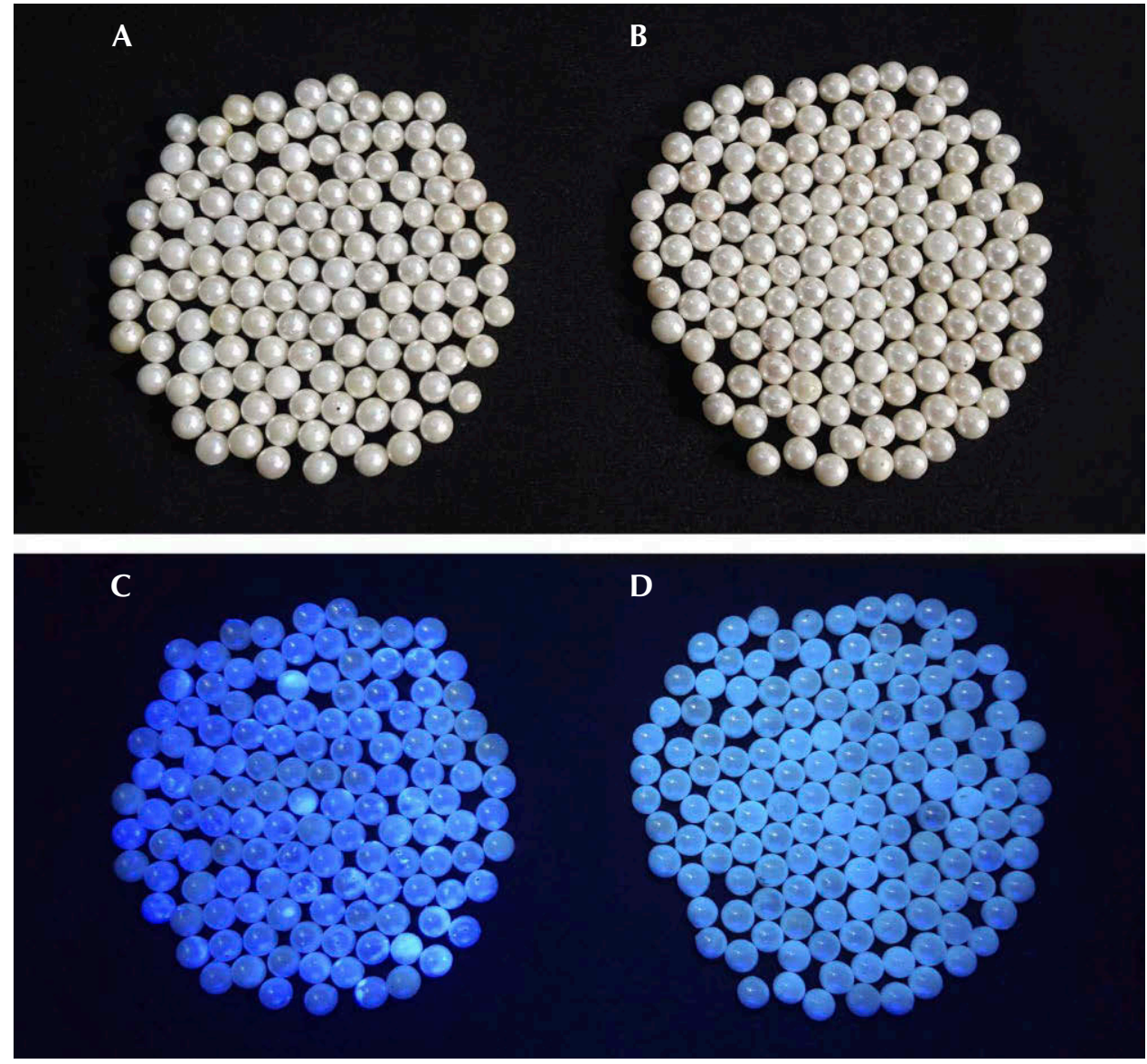

Figure 3. A group of optically brightened and bleached white akoya cultured pearls $(A)$ and a group of bleachedonly (no OBAs) akoya pearls $(B)$ under normal daylight ( $A$ and $B)$ and $L W U V$ radiation $(C$ and $D)$. The samples exposed to OBAs exhibited a more intense bluish fluorescence reaction (C). Photos by Nanthaporn Nilpetploy. 

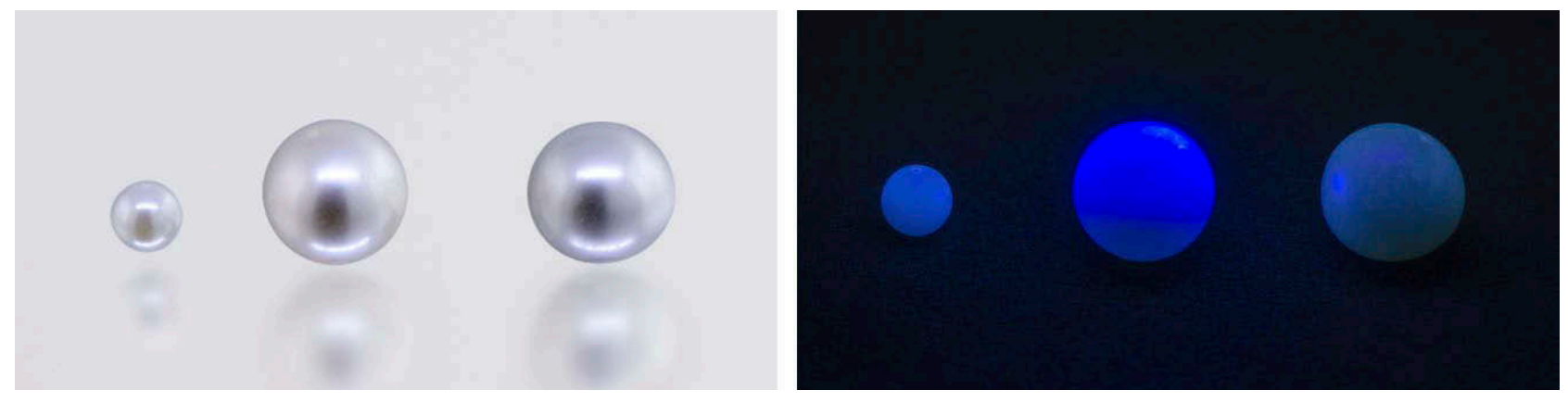

Figure 4. Three white cultured pearls_akoya (left), freshwater (middle), and South Sea (right)—shown in daylight (left photo) and exhibiting fluorescence under LWUV excitation (right photo). The freshwater pearl exhibited a distinctive blue fluorescence, which suggested it had been optically brightened. Photos by Sood Oil (Judy) Chia.

occasional stirring. It was subsequently rinsed with tap water and air-dried before further examination.

All the samples were initially examined using various standard gemological techniques, including a gemological microscope, ultraviolet-visible (UVVis) reflectance spectroscopy, and Fourier-transform (FTIR) reflectance spectroscopy. Because these tests cannot consistently identify optical brighteners, the results are not shown here. For the purpose of this particular project, a fluorescence spectroscopy unit was designed to measure pearl fluorescence with the aim of detecting OBAs. The fluorescence spectroscopy unit included a $385 \mathrm{~nm}$ UV light-emitting diode (LED) as the excitation light source, a spectrometer for monitoring the visible light region as the fluorescence sensor, and a bi-fabricated fiber-optic probe that guided the excitation light to the sample and transmitted the collected fluorescence signal from the sample to the detector. The spectrometer was configured to monitor the spectrum from 400 to $700 \mathrm{~nm}$, which covers the fluorescence range needed to analyze reactions from untreated pearls and OBAs. Two optical filters were used to isolate the pearl fluorescence from the UV LED in order to avoid the excitation light overwhelming the sensor, as well as to enhance the sensitivity of the system. A $390 \mathrm{~nm}$ short-pass filter was positioned behind the UV LED, and a $410 \mathrm{~nm}$ long-pass filter was positioned in front of the detector.

In addition to fluorescence spectroscopy, visual observation of the fluorescence reactions was performed under a conventional 5-watt LWUV (365 nm) lamp or a GIA-designed LWUV unit incorporating a narrow-band $365 \mathrm{~nm}$ UV LED source.

Figure 5. A strand of non-bleached and non-brightened akoya cultured pearls of various colors (left) and their varied fluorescence reactions under LWUV radiation (right). Photos by Sood Oil (Judy) Chia.
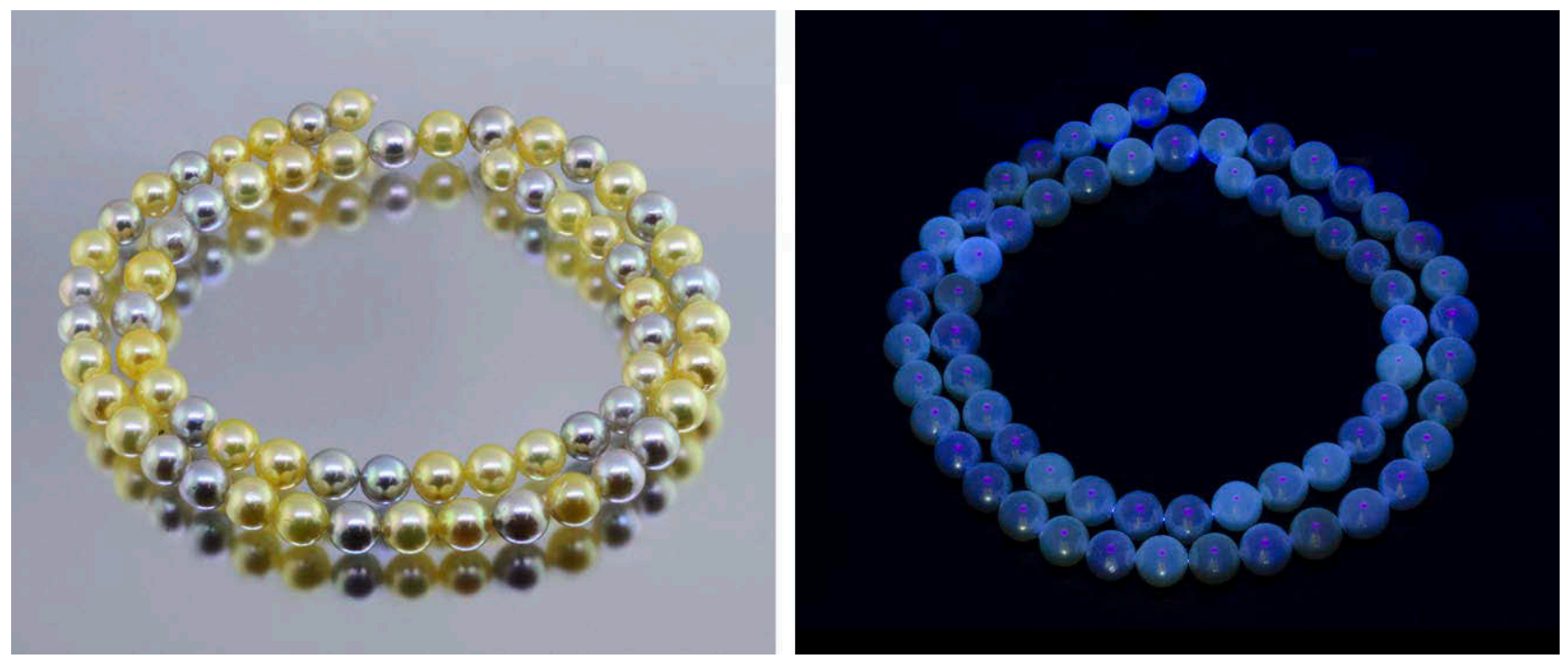


\section{FLUORESCENCE SPECTRA}

\section{RESULTS}

The two groups of akoya cultured pearls exhibited similar external appearances in terms of their size, color, shape, luster, and surface condition. The group that had been bleached and treated with OBAs appeared to be slightly whiter and more lustrous than the non-brightened group, although this could be due to the individual differences among the samples. No systematic before and after comparison on the appearances of the treated group was available. Under LWUV radiation, the group treated with OBAs showed a more consistent and stronger bluish reaction (again, see figure 3). Some of the OBA-treated pearls (especially the cream samples) showed spotty and uneven reactions under LWUV, indicating the treatment concentrated in surface areas where blemishes existed. This was confirmed by microscopy since the surface condition of the nacre was not perfect given the lower grade of akoya used in the experiment. In order to see if OBAs were detectable, fluorescence spectroscopy was applied to selected samples from each group, and the results are shown in figure 6 . All bleached-only samples tested showed broad fluorescence maxima at around 480 to $490 \mathrm{~nm}$, while the bleached and OBA-treated samples showed narrower fluorescence maxima at around 430 to 434 $\mathrm{nm}$ with an additional shoulder around $470 \mathrm{~nm}$. The

Figure 6. Fluorescence spectra of bleached and optically brightened (red) and bleached and non-brightened (blue) akoya pearls. The non-brightened pearls show a broad fluorescence maximum at around 480 to $490 \mathrm{~nm}$, whereas the OBA-treated samples exhibit a narrower fluorescence maximum at around 430 to $434 \mathrm{~nm}$ with a shoulder at around $470 \mathrm{~nm}$.

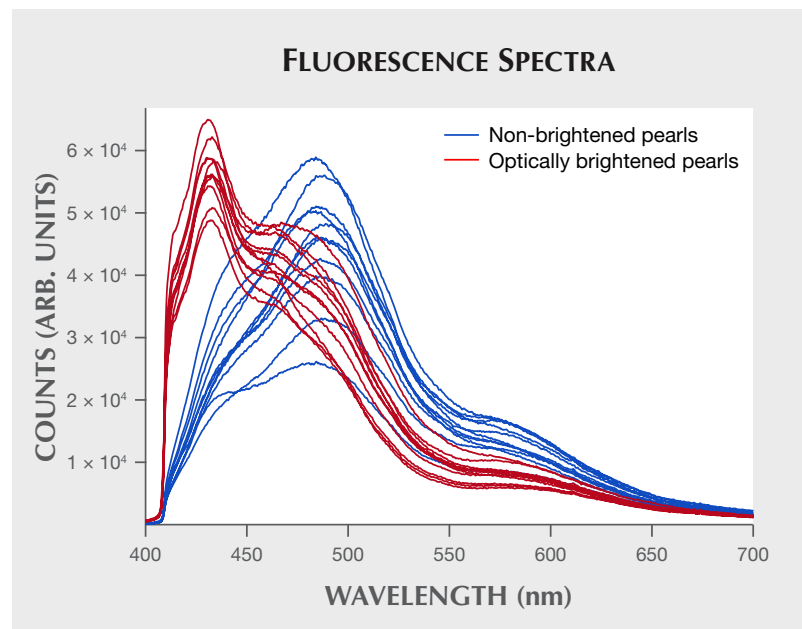

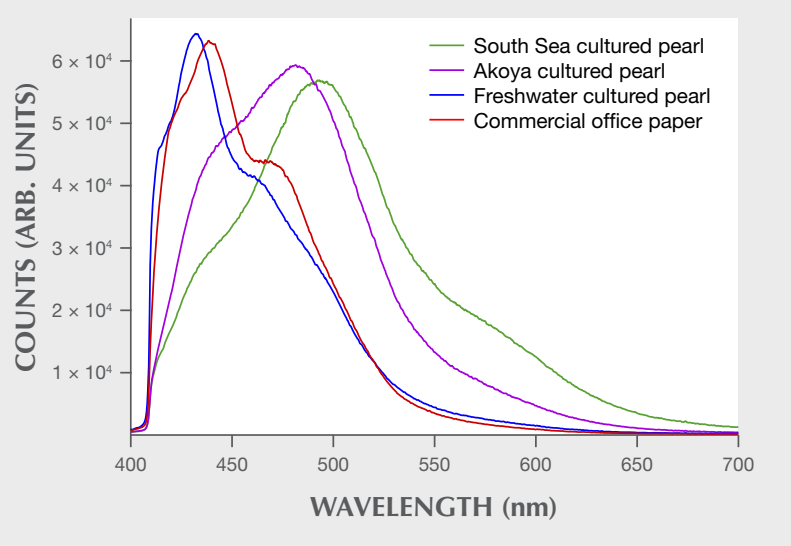

Figure 7. The fluorescence spectra of a white South Sea cultured pearl (green), white akoya cultured pearl (purple), white freshwater cultured pearl (blue), and white commercial office paper (red). The freshwater pearl tested in this study and the white paper show fluorescence results consistent with those expected for OBAs.

shifted fluorescence maxima are consistent with the fluorescence characteristics of commercially available OBAs (Lawson-Wood and Evans, 2018).

The three white cultured pearl samples from GIA's collection showed various fluorescence reactions under LWUV (figure 4, right photo). The South Sea cultured pearl showed a moderate greenish reaction and the akoya cultured pearl a slightly bluish reaction, while the freshwater pearl exhibited the most distinctive and strongest bluish reaction. These observations indicated that the South Sea cultured pearl was most likely not treated, the akoya cultured pearl was most likely traditionally processed with bleaching, and this particular freshwater pearl was also processed. Its UV fluorescence reaction looked very similar to the optically brightened akoya samples we studied. The fluorescence spectra of these three pearls confirmed these predictions (figure 7). The South Sea cultured pearl showed a fluorescence maximum at around $494 \mathrm{~nm}$, the akoya cultured pearl at around $482 \mathrm{~nm}$, and the freshwater pearl at around $432 \mathrm{~nm}$ with a shoulder at around $465 \mathrm{~nm}$. The fluorescence reaction and the spectrum of the freshwater pearl matched well with the OBA-treated akoya samples discussed earlier.

Additionally, a piece of standard commercial white office paper was tested. Its fluorescence reaction under LWUV was found to be strong blue, and its fluorescence spectrum exhibited a maximum at around 439 $\mathrm{nm}$ with a shoulder at around $474 \mathrm{~nm}$, similar to the 


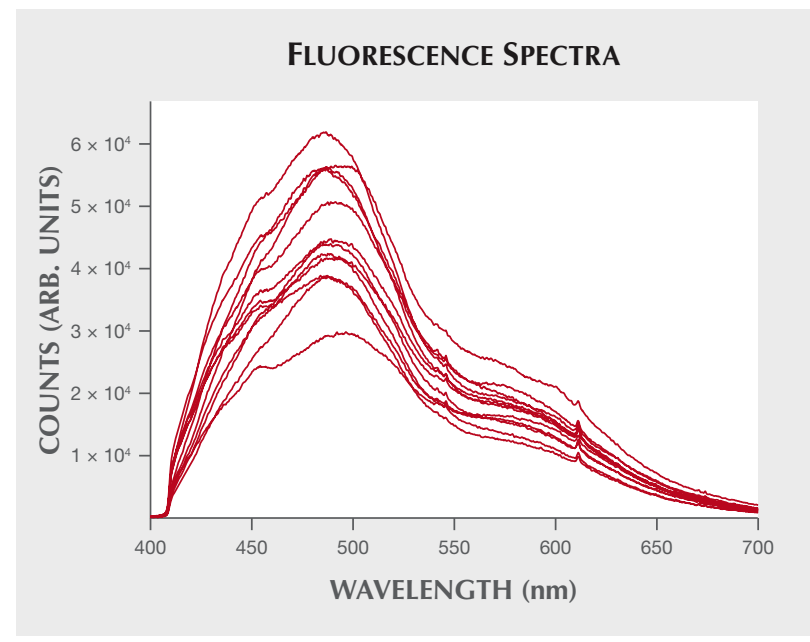

Figure 8. Fluorescence spectra of variously colored untreated akoya pearls, showing fluorescence maxima ranging from around 480 to $500 \mathrm{~nm}$. The small sharp peaks shown near 540 and $610 \mathrm{~nm}$ are due to the ambient environment (room light and computer monitor light reflection).

OBA-treated pearls studied. It was no surprise to see such features since optical brighteners are routinely applied to commercial white paper (Bajpai, 2018).

In order to verify the results, several non-bleached and non-brightened akoya cultured pearls of various colors that included cream, light yellow, silver, and light gray were tested with the same fluorescence spectroscopy method. Untreated akoya pearls can ex- hibit this same wide range of bodycolors when routine processing is not carried out (Otter et al., 2017). These pearls showed a variety of weak fluorescence reactions under LWUV radiation, with the weaker reactions the result of fluorescence quenching by their bodycolors (figure 5, right).

Spectroscopic results showed that in keeping with bleached akoya pearls, these samples exhibited a fluorescence maximum ranging between 480 and 500 $\mathrm{nm}$ (figure 8). These results and additional tests (data not shown) on GIA akoya and South Sea cultured pearls (master samples), as well as on untreated freshwater pearls of various colors from the GIA research collection, confirmed that non-brightened pearls fluoresce a weaker blue (cyan) color (480 to $500 \mathrm{~nm}$ ), while OBA-processed pearls exhibit more intense blue and violet colors (430 to $440 \mathrm{~nm}$ ).

Finally, one white South Sea cultured pearl was treated in-house using a commercial laundry product (Rit Whitener \& Brightener) containing OBAs and sodium chloride, as indicated on its label. The main ingredient listed on the manufacturer's website for this type of product is linear alkylbenzene sulfonate (CAS No. 25155-30-0). The simple method described in the Materials and Methods section resulted in an increased bluish fluorescence under LWUV, and also a slightly higher luster and whiter appearance for the pearl (figure 9). Its fluorescence changed to shorter wavelengths from around $500 \mathrm{~nm}$ to around 443 and $478 \mathrm{~nm}$ as expected, similar to the fluorescence results
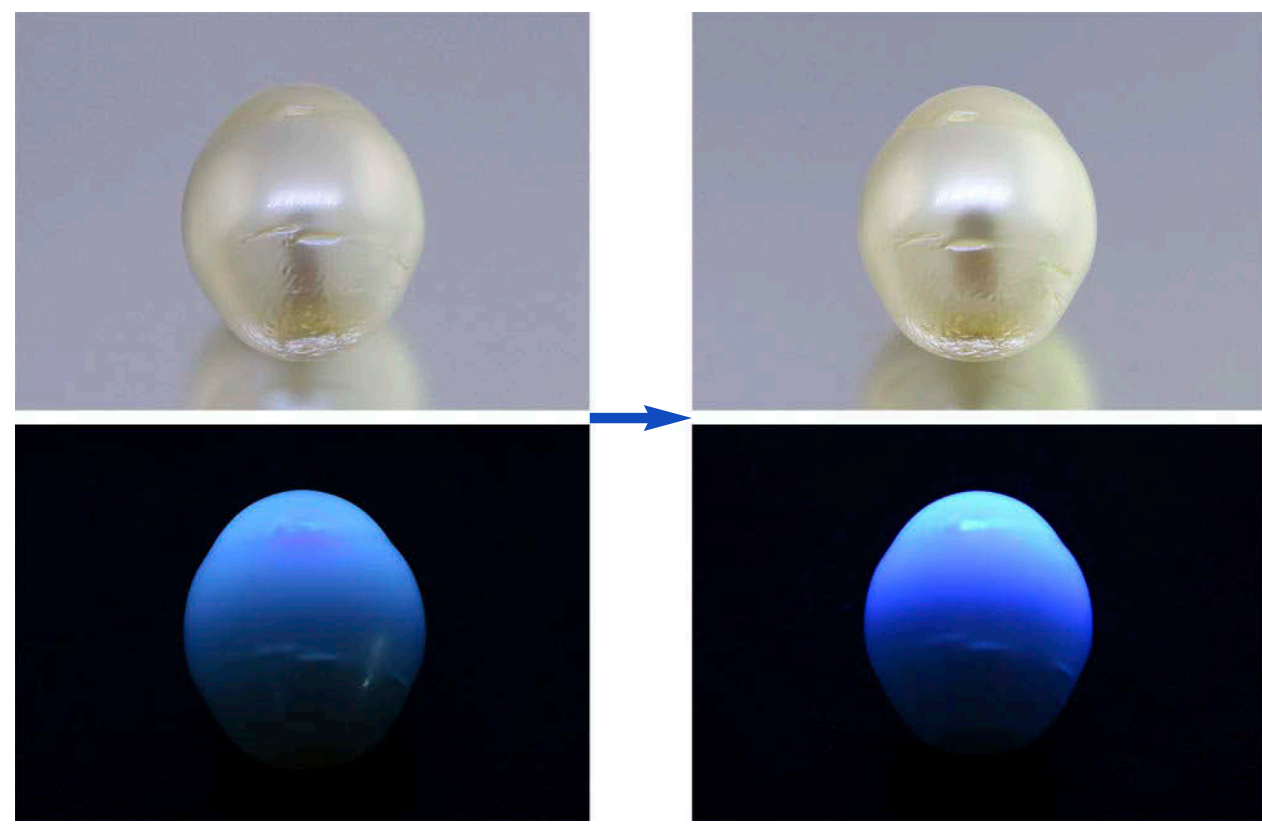

Figure 9. External appearances and fluorescence reactions under LWUV of a white South Sea cultured pearl before (left) and after (right) in-house treatment using a commercial brightener. Photos by Sood Oil (Judy) Chia. 


\section{FLUORESCENCE SPECTRA}

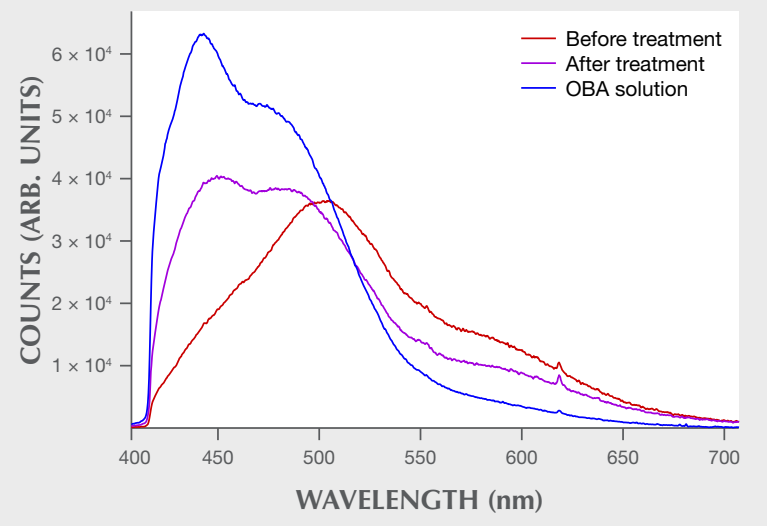

Figure 10. Fluorescence spectra of the white South Sea cultured pearl before (red) and after (purple) in-house brightening treatment. The OBA solution's spectrum is also shown in blue.

obtained for the OBA solution itself (figure 10). However, the exact OBAs applied for routine pearl treatment in the trade are most likely different from the one applied in the in-house experiment, and the actual methods are probably much more sophisticated and time sensitive. This likely explains why the fluorescence maximum of the pearl treated in-house differs from the treated samples obtained from the trade.

\section{DISCUSSION}

The application of OBAs to pearls is not new in the pearl trade, but it is sometimes not regarded as a treatment (Japan Pearl Promotion Society, 2014). OBAs are usually applied in conjunction with bleaching and are thus considered part of routine processing. Little has been discussed in the gemological literature on the identification of this process, even though optical brightening and bleaching utilize two different mechanisms to enhance the appearances of pearls. In this study, the authors focused on the identification of this process using fluorescence spectroscopy in combination with visual fluorescence observations under LWUV radiation. Both methods proved useful, but the spectroscopic method has the advantage of accurately distinguishing OBAprocessed pearls from routinely processed pearls, regardless of their colors, which might affect the visual appearance and intensity of their UV fluorescence. While bleaching may make their fluorescence inten- sities stronger, OBAs often change the fluorescence properties of the pearls more dramatically for both the fluorescence hues and intensities. These changes are consistent with the original fluorescence properties of the OBAs (shifting the fluorescence maxima to shorter wavelengths in the blue and violet range). Since there are many different types of OBAs in the market and the exact process is unknown, the effects of OBAs are not within the scope of this study, although we observed a slight increase in luster and white appearance for the South Sea cultured pearl treated in-house. With more sophisticated methods and better chemicals, these effects could be more prominent. The extent of such a process used in the trade is also not clear; the authors have heard some sources in the trade say it is used less often than it was many years ago. Similarly, the stability of such a process also requires better sample comparison and prolonged observation, which has not been fully addressed in this study.

In addition to the detection of OBAs in pearls, fluorescence spectroscopy could potentially have applications in the detection of other color treatments used on pearls (Tsai and Zhou, 2020). Furthermore, differences in the fluorescence characteristics of various types of pearls may also be used to separate pearls from different host mollusk species, as previously reported (Ju et al., 2011). While the optimal UV excitation wavelength for pearls has not been fully investigated, the excitation source used in this particular study has proved quite effective. The full potential of this advanced technique for pearls and other gemstone identification scenarios warrants greater attention.

\section{CONCLUSIONS}

This study proves that fluorescence spectroscopy, in combination with visual fluorescence observation, may provide a quick and easy way to detect the presence of different optical brighteners that have been applied to various nacreous pearls. The samples examined generally showed a strong fluorescence peak at around 430-440 due to OBAs, while non-brightened pearls usually exhibited a fluorescence peak at around $480-500 \mathrm{~nm}$. While cultured pearls may be subject to routine processes involving various types of chemical agents, it is important for the trade to be aware of this particular component and its identification criteria. 
ABOUT THE AUTHORS

Dr. Zhou is a senior manager of pearl identification at GIA in New York. Dr. Tsai is a research scientist at GIA in New Jersey. Mr. Sturman is a senior manager, Ms. Nilpetploy is a senior staff gemologist, Ms. Manustrong is a staff gemologist, and Ms. Lawanwong is an analytics technician, in GIA Bangkok's pearl department.

\section{ACKNOWLEDGMENTS}

The authors would like to thank Mr. Devchand Chodhry, managing director of Orient Pearl (Bangkok) Ltd. for providing some samples in this study to GIA's Bangkok laboratory.

\section{REFERENCES}

Bajpai P. (2018) Optical properties of paper. Chapter 11 in Biermann's Handbook of Pulp and Paper (3rd ed.). Elsevier, pp. 237-271, https://doi.org/10.1016/B978-0-12-814238-7.00011-8

Esteves M.F., Noronha A.C., Marinho R.M. (2004) Optical brighteners effect on white and coloured textiles. World Textile Conference - 4th AUTEX Conference, Roubaix, June 22-24.

Japan Pearl Promotion Society (2014) Pearl Standard (English version), Revised Version, p 21. http://jp-pearl.com/wpcontent/uploads/2017/09/2014版真珠スタンタート :\%EF\%BC\% 88英語\%EF\%BC\% 89.pdf

Ju M.J., Lee S.J., Kim Y., Shin J.G., Kim H.Y., Lim Y., Yasuno Y., Lee B.H. (2011) Multimodal analysis of pearls and pearl treatments by using optical coherence tomography and fluorescence spectroscopy. Optics Express, Vol. 19, No. 7, pp. 6420-6432.

Lakowicz J.R. (2006) Principles of Fluorescence Spectroscopy, 3rd ed. Springer US, pp. 5-7.

Lanter J. (1966) Properties and evaluation of fluorescent brightening agents. Journal of the Society of Dyers and Colourists. Vol. 82, No. 4, pp. 125-132.

Lawson-Wood K., Evans K. (2018) Measurement of optical bright- ening agents in samples using the FL 6500 fluorescence spectrometer and solid sample holder. Application Brief, Fluorescence Spectroscopy, PerkinElmer, Inc., Seer Green, UK.

Leaver I.H., Milligan B. (1984) Fluorescent whitening agents-a survey (1974-82). Dyes and Pigments, Vol. 5, No. 2, pp. 109-144.

Otter L.M., Agbaje O.B.A., Huong L.T-T., Häger T., Jacob D.E. (2017) Akoya cultured pearl farming in eastern Australia. $G \uplus G$, Vol. 53, No. 4, pp. 423-437, http://dx.doi.org/10.5741/GEMS.53.4.423

Shor R. (2007) From single source to global free market: The transformation of the cultured pearl industry. $G \uplus G$, Vol. 43, No. 3, pp. 200-226, http://dx.doi.org/10.5741/GEMS.43.3.200

Song H.C., Fan Y. (1999) Bleaching technique for pearls (in Chinese). Journal of Fisheries of China, Vol. 4, pp. 398-402.

Tsai T.-H., D'Haenens-Johansson U.F.S. (2019) Gemstone screening and identification using fluorescence spectroscopy, Frontiers in Optics + Laser Science APS/DLS, OSA Technical Digest (Optical Society of America, 2019), paper JTu3A.109, https://doi.org/10.1364/FIO.2019.JTu3A.109

Tsai T.-H., Zhou C. (2020) Lab Notes: Fluorescence spectroscopy for colored pearl treatment screening. $G \uplus G$, Vol. 56, No. 1, pp. 136-137.

\section{For online access to all issues of GEMS \& GEMOLOGY from 1934 to the present, visit:}

